\title{
Protein inhibitor of activated STAT x $\alpha$ depresses cyclin D and cyclin D kinase, and contributes to the inhibition of osteosarcoma cell progression
}

\author{
JUNJIE WANG $^{1}$, JIANGDONG NI ${ }^{1}$, SHUAI YI $^{2}$, DEYE SONG $^{1}$ and MULIANG DING ${ }^{1}$ \\ Departments of ${ }^{1}$ Orthopedics and ${ }^{2}$ Ophthalmology, Second Xiangya Hospital, \\ Central South University, Changsha, Hunan 410011, P.R. China
}

Received December 24, 2014; Accepted September 18, 2015

DOI: $10.3892 / \mathrm{mmr} .2015 .4705$

\begin{abstract}
Previous studies have shown that protein inhibitor of activated STAT (PIAs)x $\alpha$ is crucial in protein sumoylation and is associated with cancer cell progression. However, the mechanism underlying the inhibitory effect on cancer cells, which may assist in developing novel treatment strategies in cancer remains to be elucidated. In present study, the expression levels of PIAsx $\alpha$ from tissue samples of osteosarcoma and adjacent tissues from 25 patients were analyzed using reverse transcription-quantitative polymerase chain reaction, western blot and immunohistochemical analyses. In addition, techniques using an overexpression vector and small interfering (si)RNAs were used to examine the effect of PIAsx $\alpha$ on osteosarcoma cells. Finally, using xenograft U2-OS osteosarcoma cells overexpressing PIAsx $\alpha$, the effect of PIAsx $\alpha$ on osteosarcoma formation was determined. The results revealed low expression of PIAsx $\alpha$ in osteosarcoma tissues. In addition, following overexpression of PIAsx $\alpha$, the apoptotic rates were significantly increased. The rate of G2/M arrest was at the highest level in the overexpression group, compared with other groups assessed. Furthermore, the expression levels of cyclin D1 and cyclin D3 were inhibited following PIAsx $\alpha$ increase, indicating the repressive effects of PIAsx $\alpha$ on cell cycle. Accordingly, cyclin D kinase (CDK) genes, including CDK4, CDK6 and CDK8, increased markedly following treatment with PIAsx $\alpha$ siRNAs. The expression levels of CDK4, CDK6 and CDK8 decreased significantly in the overexpression group, compared to the other groups. Furthermore, high expression levels of PIAsx $\alpha$ inhibited tumor formation in a nude mouse model. Taken together, these findings provide evidence for the effects of PIAsx $\alpha$ and its mechanism on osteosarcoma
\end{abstract}

Correspondence to: Professor Jiangdong Ni, Department of Orthopedics, Second Xiangya Hospital, Central South University, 139 Renmin Road, Changsha, Hunan 410011, P.R. China

E-mail: nijiangdong_doc@126.com

Key words: protein inhibitor of activated STAT x $\alpha$, osteosarcoma, cyclin D, cyclin D kinase, sumoylation progression, which offers novel insight into sumoylation and the cell cycle in osteosarcoma.

\section{Introduction}

Sumoylation, a post-translation modifier, is involved in various biological processes and, as one of the most intensively investigated fields in cancer pathogenesis, has been suggested to modify proteins by attaching small ubiquitin-like modifier (SUMO), which affects function of the target proteins in organismal development $(1,2)$. At present, three paralogs of SUMO have been found, including SUMO-1, SUMO-2 and SUMO-3 $(3,4)$. The latest reports indicate a novel paralog, SUMO-4, however, no evidence has been reported to demonstrate whether the SUMO-4 protein is an endogenously expressed protein in cells $(5,6)$. SUMO-specific protease 1 (SENP1), is one of the members of de-sumoylation protease, which is curial in transcriptional regulation and hypoxia signaling (7). Further investigations have suggested that SENP1 is associated with the progression of prostate cancer $(8,9)$. In addition, SUMO-1 can alter the activation of promyelocytic leukemia protein oncogenic domains (10). Thus, sumoylation has been regarded as an important pathway for disease pathogenesis, which merits its further investigation.

Until now, substantial evidence indicates that SUMO modification is involved in tumorigenesis $(11,12)$. The levels of ubiquitin-conjugating enzyme (UBC)9, one of homologous of UBCs, have been found to be increased in several types of cancer (13). In addition, UBC9 promotes tumor cell growth (14). Protein inhibitor of activated STAT (PIAs)3, one of the members of the SUMO E3 pathway, is also increased in several types of cancer, including lung cancer, pancreatic cancer, colorectal cancer and hepatocellular carcinoma (15-17). Furthermore, sumoylation may regulate tumor suppressor proteins, including p53,p63,p73 and retinoblastoma protein (12). The $\mathrm{p} 53$ protein is stimulated by a degradative pathway, which can be modified by SUMO-1 sumoylation at the K386 site in the C-terminus (18). Thus, SUMO-1 regulates the crucial pathway of cancer progression in organisms or cells, rendering it a potential target for therapeutic modulators.

PIAs contain PIAs1, PIAs3, PIAs1, PIAs3, PIAs3L and PIAsy, as well as PIAsx $\alpha$ and $\beta$ splice variants (19). These 
proteins are predominantly expressed in the nucleus and are characterized by an N-terminal SAP and C-terminal SP-RING domain (19). A previous study showed that PIAsx $\alpha$ can stimulate E3 ligases in sumoylation (20). PIAsx $\alpha$ has also been shown to act as a ligase of SUMO E3, and suppresses tumor development by promoting the expression of phosphatase and tensin homologue deleted on chromosome (PTEN) (21). These findings suggest that PIAsx $\alpha$ is a novel SUMO E3 ligase in the PTEN-associated cancer progression pathway.

In tumor cells, cell proliferation and division are regulated by key regulators of the cell cycle, including cyclin D kinase (CDK) and cyclin D proteins (22). If the expression of these genes are disrupted, the cell cycle is arrested or delayed at checkpoints, and results in the inhibition of cell proliferation. In addition, $\mathrm{CDK}$ and cyclin D proteins stabilize the genome in the G1 phase, which assists genome duplication in this process (23). Thus, the dysregulation of CDKs and cyclins trigger disordered cell proliferation and cell division, which are found in cancer cells.

In present study, an overexpression vector and siRNAs were used to understand the effects of PIAsxa on U2-OS osteosarcoma cells. In addition, the progression of the U2-OS cell line was confirmed using flow cytometry. The expression levels of CDKs and cyclin D were detected following transfection to induce overexpression or with siRNAs, and treatment to rescue the effects of overexpression and siRNA transfection, in U2-OS cells. The results of these investigations may elucidate the effects of PIAsx $\alpha$ and its mechanism on the progression of osteosarcoma.

\section{Materials and methods}

Patients. Tissue samples from patients with osteosarcoma were collected from the Second Hospital of Xiangya, Central South University (Changsha, China). The experiments were performed, according to guidelines and following approval by Xiangya School of Medicine Research Ethics Committee, Central South University. Written informed consent was obtained from the patients, according to the Declaration of Helsinki (24). The clinical parameters of the patients, including age, gender and histological examination of the state of invasiveness were also collected. The osteosarcoma and adjacent tissues samples were obtained from 25 patients diagnosed with TNM stage III osteosarcoma. These patients had not undergone radical prostatectomy or any previous treatment.

Cell line and culture. The U2-OS osteosarcoma cell line was provided by the Department of Orthopedic Surgery, Xiangya Hospital of Central South University. The cells were seeded into 24-well plates at a density of $1 \times 10^{6}$ cells per well. The cells were cultured in Dulbecco's modified Eagle's medium (DMEM; Sigma-Aldrich, St. Louis, MO, USA) with $10 \%$ bovine serum (Sigma-Aldrich) in a $37^{\circ} \mathrm{C}$ humidified incubator. The concentration of $\mathrm{CO}_{2}$ in the humidified incubator was $5 \%$. Cell viability was confirmed using trypan blue staining, and visualized using a Nikon Eclipse E400 microscope (Nikon, Tokyo, Japan).

Transfection of PIAsxa vectors. To analysis the effect of PIAsx $\alpha$ on osteosarcoma cell progression, a PIAsx $\alpha$ overexpression vector was constructed. The overexpression vector was constructed using a pcDNA3.1(t) plasmid (Invitrogen; Thermo Fisher Scientific, Inc., Waltham, MA, USA). The full-length coding sequence of PIAsx $\alpha$ was obtained from GenBank (www.ncbi.nlm.nih.gov/genbank/), and the following primers were designed to obtain the coding sequence with $X b a \mathrm{I}$ and HindIII sites: Forward 5'-TTGTCTAGAATGGCGGATTTCGAAGAGTT-3' and reverse 5'-AAGCTTTCACTGTTGCACAGTATCAGA-3'. The fragments were cloned using a cDNA library from adjacent tissues. A T100 ${ }^{\mathrm{TM}}$ thermal cycler was used (Bio-Rad Laboratories, Inc., Richmond, CA, USA). The PCR reaction comprised 30 cycles of $94^{\circ} \mathrm{C}$ for $30 \mathrm{sec}, 52^{\circ} \mathrm{C}$ for $30 \mathrm{sec}$ and $72^{\circ} \mathrm{C}$ for $3 \mathrm{~min}$. Subsequently, the PCR product was digested with $X b a I$ and HindIII (Shanghai Sangon Biotech Co., Ltd., Shanghai, China). The digested PCR fragments were inserted into the pcDNA3.1(t) plasmid (Invitrogen; Thermo Fisher Scientific, Inc.) using T4 DNA Ligase (Shanghai Sangon Biotech Co., Ltd.). Subsequently, the vector was transfected into the U2-OS cells using Lipofectamine ${ }^{\mathrm{TM}} 2000$ (Invitrogen; Thermo Fisher Scientific, Inc.).

Small interfering (si)RNAs of PIAsx $\alpha$ and the GAPDH control were constructed using a Silencer siRNA Construction kit (Ambion; Thermo Fisher Scientific, Inc.), according to a previous report (21). The siRNA sequence was 5'-GUGGAAGGCAACGAGUGGAUU-3, which was synthesize by Shanghai Sangon Biotech Co., Ltd. The target sequence was 5'-AATCCACTCGTTGCCTTCCAC-3'. The transfection protocol was performed, as described previously.

The cells cultured in 12-well plates $\left(1 \times 10^{6}\right)$ were randomly divided into five groups: Untreated control group; PIAsx $\alpha$ interference group (20 $\mu \mathrm{M}$ PIAsx $\alpha$ siRNA); PIAsx $\alpha$ overexpression group (1 $\mu \mathrm{g}$ PIAsx $\alpha$ pcDNA3.1(t) vector transfection); PIAsx $\alpha$ interference rescue group ( $20 \mu \mathrm{M}$ siRNAs for $24 \mathrm{~h}$ following $1 \mu \mathrm{g}$ pcDNA3.1(t) PIAsx $\alpha$ vector transfection for $24 \mathrm{~h}$ ); and PIAsx $\alpha$ overexpression rescue group (1 $\mu \mathrm{g}$ pcDNA3.1(t) PIAsx $\alpha$ vector transfection for $24 \mathrm{~h}$ following $20 \mu \mathrm{M}$ siRNAs for $24 \mathrm{~h}$ ). Each group was treated in five independent parallel experiments. Following the respective treatments, analyses of the cells were performed.

Flow cytometry. The cells were first fixed in $70 \%$ ethanol overnight and resuspended in propidium iodide $(50 \mu \mathrm{g} / \mathrm{ml}$; Sigma-Aldrich). The DNA content was measured using a flow cytometer (FACScan; BD Biosciences, Franklin Lakes, NJ, USA). The cells were treated with $0.5 \mu \mathrm{g} / \mathrm{ml}$ annexin $\mathrm{V}$-phycoerythrin and $0.5 \mu \mathrm{g} / \mathrm{ml} 7$-aminoactinomycin D (7-ADD; BD Biosciences) for $15 \mathrm{~min}$ at room temperature. Cell death was subsequently calculated based on positive signals of annexin V, 7-AAD, or the two combined.

Reverse transcription-quantitative polymerase chain reaction $(R T-q P C R)$. The mRNA expression levels of the assessed genes, including cyclin D and CDK, were detected using RT-qPCR. The total RNAs of the cells were extracted using TRIzol reagent (Invitrogen; Thermo Fisher Scientific, Inc.), according to the manufacture's protocol. Subsequently, $1 \mu \mathrm{g}$ of the total RNAs was used to synthesize the first chain of cDNA, which was constructed using a Takara cDNA Library Construction kit (Takara Biotechnolohy Co., Ltd., Dalian, 
China). Subsequently, the expression levels of cyclin D1, cyclin D3, CDK4, CDK6 and CDK8 were assayed on a 7500 Real-Time PCR system Applied Biosystems; Thermo Fisher Scientific, Inc.). The reaction mixture contained $1 \mu \mathrm{l}$ cDNA, $10 \mu 1 \mathrm{X}$ reaction buffer. and $0.1 \mathrm{mM}$ forward and reserve primers, respectively. The primers for the cyclin D1, cyclin D3, CDK4, CDK6 and CDK8 genes were designed, as in a previous study (25), with the following sequences: cyclin D1, forward 5'-TCG TTG CCC TCT GTG CCA CA-3' and reverse: 5'-AGG CAG TCC GGG TCA CAC TT-3'; cyclin D3, forward 5'-TGG TCC TAG GGA AGC TCA AGT G-3' and reverse 5'-CTG TAG CAC AGA GGG CCA AAA-3'; CDK4, forward 5'-GCT ACC ACT CGA TAT GAA CCC GTG GCT GAA-3' and reverse 5'-GGT GCT TTG TCC AGG TAT GTC CGT AGG TCC-3'; CDK6, forward 5'-ATC TAT AGT TTC CAG ATG GCT CTA ACC-3' and reverse 5'-ACA AAC TTC TCA ATT GGT TGG GCA GAT TT-3'; CDK8, forward 5'-ATG GAC TAT GAC TTT AAA GTG AAG CTG AGC AGC GA-3' and reverse 5'-ATC AGC ATG AGA CAG AAA CAC CTT TTG AAG-3'; and GAPDH, forward 5'-AAC CCA GAA GAC TGT GGA TGG-3' and reverse 5'-GGA GAC AAC CTG GTC CTC AG-3'. The PCR conditions were as follows: $95^{\circ} \mathrm{C}$ for $10 \mathrm{~min}$, followed by 40 cycles at $95^{\circ} \mathrm{C}$ for $15 \mathrm{sec}$ and $60^{\circ} \mathrm{C}$ for $45 \mathrm{sec}$. The mRNA relative expression levels were analyzed using the $2^{-\Delta \Delta \mathrm{Cq}}$ method (26).

Western blot analysis. The protein expression levels of the assessed genes, including cyclin D and CDK, were detected using western blot analysis. The cells were first dissociated using radioimmunoprecipitation assay buffer (Santa Cruz Biotechnology, Inc., Santa Cruz, CA, USA), and centrifugation was performed at $20,000 \mathrm{x}$ g for $15 \mathrm{~min}$ at $4^{\circ} \mathrm{C}$. to isolate the protein in the cells. The proteins were quantified using a Bradford kit (Beyotime Institute of Biotechnology), according to the manufacturer's protocol. Subsequently, $10 \mu \mathrm{g}$ protein of each sample was separated on $10 \%$ SDS-PAGE gels (Bio Rad Laboratories, Inc., Hercules, CA, USA) and then transferred onto polyvinylidene fluoride membranes (Santa Cruz Biotechnology, Inc.). Following blocking with $4 \%$ non-fat milk, the membranes were incubated at $4^{\circ} \mathrm{C}$ overnight with following monoclonal rabbit primary antibodies: anti-PIAsx $\alpha$ (cat. no. ab50226; Abcam, Cambridge, MA, USA), anti-cyclin D1 (cat. no. ab7958; Abcam), anti-cyclin D3 (cat. no. ab112034; Abcam), anti-CDK4 (cat. no. ab7955; Abcam), anti-CDK6 (cat. no. ab151247; Abcam), anti-CDK8 (cat. no. ab1239401 Abcam) and anti-GAPDH (cat. no. ab9485; Abcam). The membranes were then washed three times using $1 \mathrm{X}$ phosphate-buffered saline with $0.05 \%$ Tween-20, and incubated with secondary antibody (goat anti-rabbit IgG; cat. no. ab6721; Abcam) at room temperature for $30 \mathrm{~min}$. The signals were detected using chemiluminescent substrate (ECL Plus; GE Healthcare Life Sciences, Chalfont, UK). The primary antibodies used in the present study were monoclonal antibodies of recombination human proteins produced from rabbit: anti-PIAsxa (cat. no. ab50226; Abcam), anti-cyclin D1 (cat. no. ab7958; Abcam), anti-cyclin D3 (cat. no. ab112034; Abcam), anti-CDK4 (cat. no. ab7955; Abcam), anti-CDK6 (cat. no. ab151247; Abcam), anti-CDK8 (cat. no. ab1239401 Abcam) and anti-GAPDH (cat. no. ab9485; Abcam). Quantification of protein expression levels was performed using Gel-Pro Analyzer 4.0 (Media Cybernetics, Inc., Silver Spring, MD, USA). The mean band intensity of three repeats was determined, and the relative intensity of each blot was calculated as a percentage of the blot.

Immunohistochemistry. The tissues were fixed in $10 \%$ formalin overnight and were prepared as $10 \mu \mathrm{m}$ thick tissue sections. Following de-waxing, the samples were stained using primary antibodies at $4^{\circ} \mathrm{C}$ overnight and secondary antibodies at room temperature for $30 \mathrm{~min}$, as described above. A horseradish peroxidase complex and diaminobenzidine were used to visualize the signals (Beyotime Institute of Biotechnology, Haimen, China). The slides were mounted, according to the following criteria: 0 , no staining; 1 , partial staining around cells; 2 , strong staining of cells. For each sample, 10 visual fields were randomly selected. Images were obtained using an OLYMPUS CX31 microscope (Olympus Corporation, Tokyo, Japan).

The treated U2-OS osteosarcoma cells were first transferred onto sterile glass cover slips and then fixed with 4\% paraformaldehyde (Shanghai Sangon Biotech Co., Ltd.). Following blocking with $5 \%$ fetal bovine serum, the cells were incubated with primary antibodies, as described above, for $1 \mathrm{~h}$ at $37^{\circ} \mathrm{C}$. Following incubation with secondary antibodies with fluorescein isothiocyanate-goat anti-rabbit IgG (Santa Cruz Biotechnology, Inc.), the signals of the proteins were detected using a fluorescence microscope (Nikon TMS; Nikon Corporation, Tokyo, Japan).

Tumor formation in a nude mouse model. To determine the function of PIAsx $\alpha$ in osteosarcoma cells in vivo, 5-week-old nude mice were used as model of tumor formation. The animal experiments performed were approved by Xiangya School of Medicine Research Ethics Committee, Central South University. The mice were maintained under specific pathogen-free conditions in a $12 \mathrm{~h}$ light/dark cycle with a room temperature of $24 \pm 1^{\circ} \mathrm{C}$. All mice were provided with rodent chow (Prolab RMH 3000; PMI LabDiet, Richmond, IN, USA) and distilled deionized water. Treatment groups were housed separately. A total of 20 female nude mice were randomly divided into two groups (10 mice in each group). The two groups were injected with U2-OS cells and cells overexpressing PIAsx $\alpha 24 \mathrm{~h}$ following transfection, respectively. For each individual, 20,000 cells in $1 \mathrm{ml}$ DMEM were injected on the dorsal side every 3 days for a total of 21 days. The tumor volume was calculated as follows: Volume $\left(\mathrm{mm}^{3}\right)=\left(\right.$ length $\mathrm{x}$ width $\left.^{2}\right) / 2$. The length and width of the tumor was measured using vernier calipers without anesthesia or surgery. The gene expression levels were determined at the end of the 21 day period. Following the experiments, the mice were anesthetized and sacrificed under xylazine $(10 \mathrm{mg} / \mathrm{kg}$, intraperitoneally; Invitrogen; Thermo Fisher Scientific, Inc.), and the tissues were collected for examination.

Statistical analysis. The data are presented as the mean \pm standard deviation. All data were statistically analyzed using SPSS 17.0 software (SPSS, Inc., Chicago, IL, USA). Significant differences in expression were confirmed using one-way analysis of variance. $\mathrm{P}<0.05$ was considered to indicate a statistically significant difference. 
A

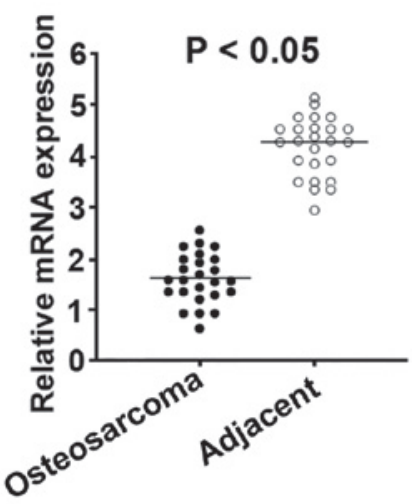

C

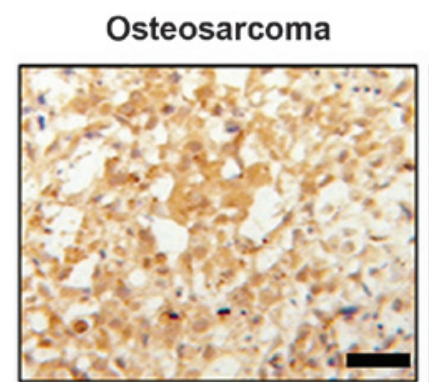

B

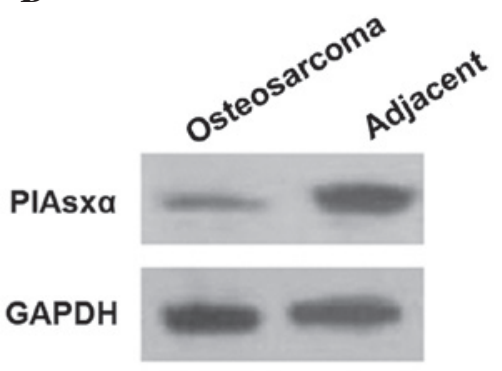

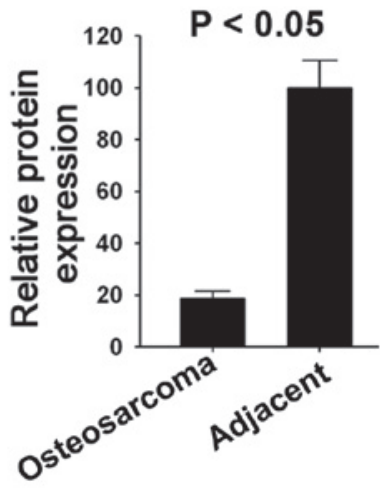

Adjacent

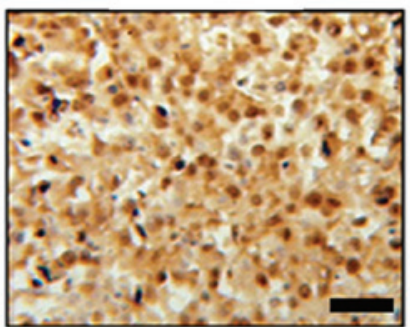

Figure 1. Expression of PIAsx $\alpha$ is reduced in osteosarcoma. (A) mRNA expression levels of PIAsx $\alpha$ in osteosarcoma and adjacent tissues were detected using reverse transcription-quantitative polymerase chain reaction analysis. (B) Western blot analysis results showed lower expression levels of PIAsx $\alpha$ in osteosarcoma tissues, compared with adjacent normal tissues. (C) Immunohistochemical localization of PIAsx $\alpha$ in the osteosarcoma tissues and adjacent tissues Expression was higher in the adjacent tissues, compared with the osteosarcoma tissues. (Scale bar=200 $\mu$ m). PIAsx $\alpha$, protein inhibitor of activated STAT x $\alpha$.

\section{Results}

Expression of PIAsxa is reduced in osteosarcoma. The mRNA expression levels of PIAsx $\alpha$ in osteosarcoma were significantly lower in the osteosarcoma tissues, compared with the adjacent normal tissues, which were determined using RT-qPCR (Fig. 1A). Similarly, the protein expression levels of PIAsx $\alpha$ were lower in the osteosarcoma tissues, compared with the adjacent tissues (Fig. 1B and C).

PIAsxa induces apoptosis in the U2-OS cell line. To understand the effects of PIAsxa on osteosarcoma cell progression, the present study used overexpression and interference techniques via an overexpression vector and siRNAs. The results of the flow cytometric analysis revealed that the levels of apoptosis were highest in the overexpression group (Fig. 2A and B). In addition, the rate of $\mathrm{G} 2 / \mathrm{M}$ arrest was also highest in the overexpression group, compared with the rates in the other groups assessed (Fig. 2C).

Overexpression and interference of PIAsxa in the U2-OS cell line. The mRNA and protein expression levels in the U2-OS cell line following overexpression and interference of PIAsx $\alpha$ were detected using RT-qPCR, and western blot and immunofluorescence analyses. The mRNA levels of PIAsx $\alpha$ in the U2-OS cells following overexpression were the highest among all the groups, whereas the PIAsx $\alpha$ interference group showed the lowest levels of expression. The control, overexpression rescue and interference rescue groups had the median expression levels among the groups (Fig. 3A), and the protein expression levels were similar. The overexpression group and interference group showed the highest and lowest protein expression levels among all the groups in the western blot and immunofluorescence analyses, respectively (Fig. 3B and C). These results indicated that the induced overexpression and interference of PIAsx $\alpha$ in the U2-OS cell line were successful.

PIAsxa downregulates the expression levels of cyclin $D$ and $C D K$ in U2-OS cells. The present study aimed to further investigate the inhibitory effects of PIAsx $\alpha$ on U2-OS cells by examine the mechanism underlying these inhibitory effects. The results of the present showed that, following overexpression of PIAsx $\alpha$, the expression levels of cyclin D1 and cyclin D3 were inhibited. By contrast, the expression levels of cyclin D1 and cyclin D3 following PIAsx $\alpha$ interference were markedly increased, compared with the control group. Similarly, the CDK genes including CDK4, CDK6 and CDK8, increased markedly following PIAsx $\alpha$ interference. By contrast, the expression levels of CDK4, CDK6 and CDK8 decreased significantly in the overexpression group, compared with the other groups (Fig. 4A and B). These results demonstrated the inhibitory effects of PIAsx $\alpha$ on the cyclin D and CDK pathways in the osteosarcoma cells.

PIAsxa inhibits tumor formation in nude mice. The xenograft inducing overexpression of PIAsx $\alpha$ in the U2-OS cells depressed tumor formation, compared with the untreated U2-OS cells (Fig. 5A). In addition, the mRNA 1 and protein expression levels of cyclin D and CDK were depressed by the increased expression of PIAsx $\alpha$ (Fig. 5B and C), indicating that the PIAsx $\alpha$ repressed these genes in the nude mouse tumor model. 
A
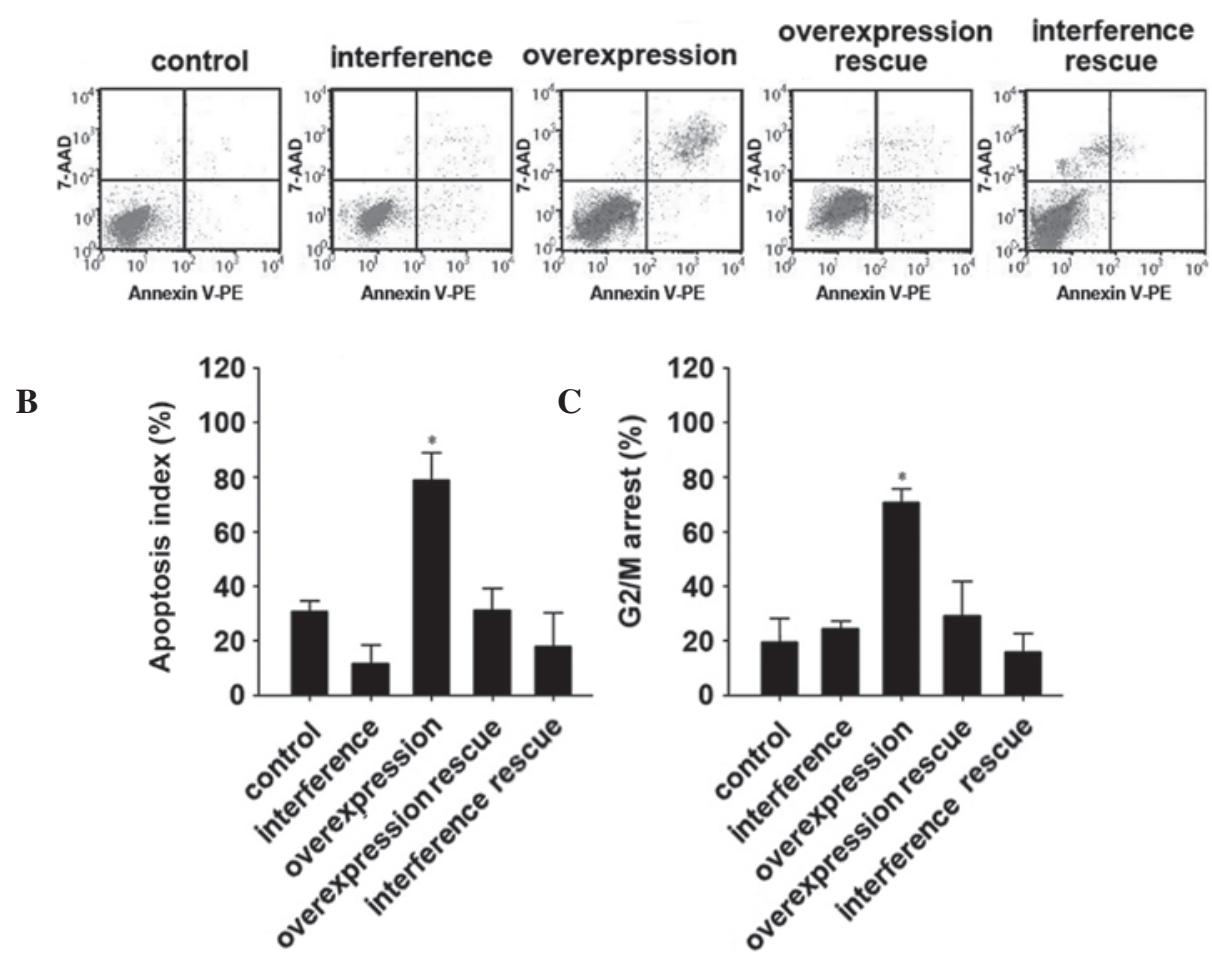

Figure 2. Effects of PIAsx $\alpha$ overexpression and interference on osteosarcoma cell progression. (A) Flow cytometric analyses of the control, interference, overexpression, overexpression rescue and interference rescue groups. (B) Apoptosis indices in the control, interference, overexpression, overexpression rescue and interference rescue groups. (C) Rates of G2/M arrest in the control, interference, overexpression, overexpression rescue and interference rescue groups. Data are presented as the mean \pm standard deviation. "P<0.05, compared with the control. PIAsx $\alpha$, protein inhibitor of activated STAT x $\alpha$; 7-ADD, 7-aminoactinomycin D; PE, phycoerythrin.

$\mathbf{A}$

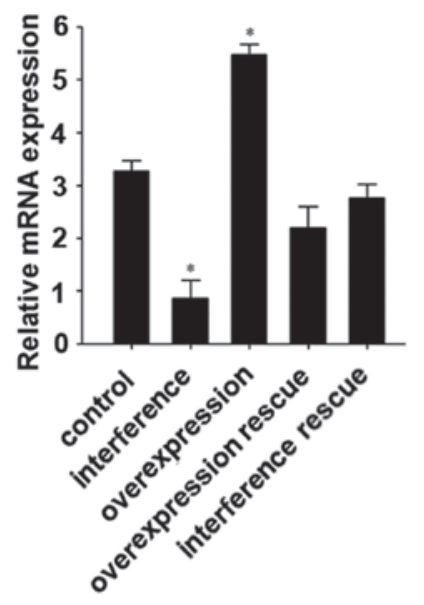

B

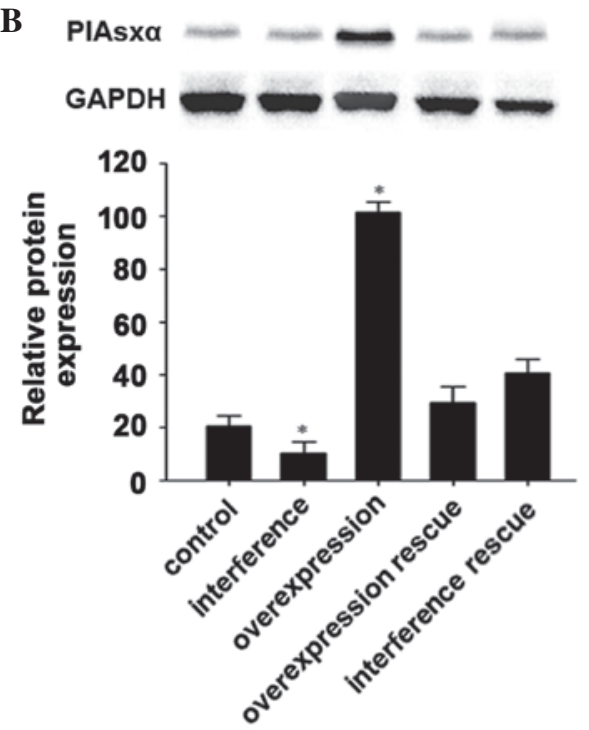

C
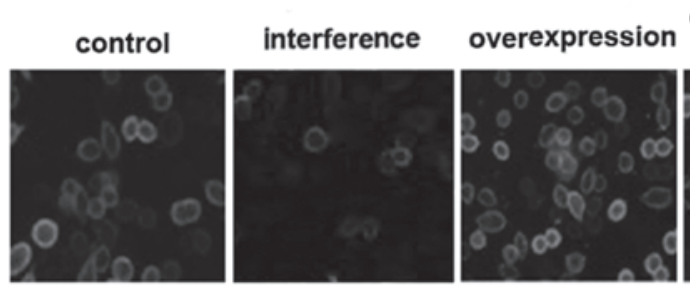

$$
\text { overe }
$$

verexpression

interference

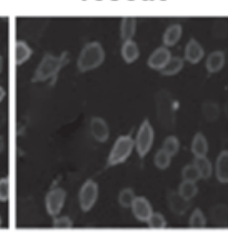

rescue

Figure 3. Overexpression and interference of PIAsx $\alpha$ in osteosarcoma cells. (A) Reverse transcription-quantitative polymerase chain reaction analysis was used to determine the mRNA expression levels in the control, interference, overexpression, overexpression rescue and interference rescue groups. Data are presented as the mean \pm standard deviation. ${ }^{*} \mathrm{P}<0.05$, compared with the control. (B) Western blot analysis was used to determine the protein expression levels in the control, interference, overexpression, overexpression rescue and interference rescue groups. (C) Immunofluorescence analyses of PIAsx $\alpha$ in the different groups (magnification, x600). PIAsx $\alpha$, protein inhibitor of activated STAT x $\alpha$. 


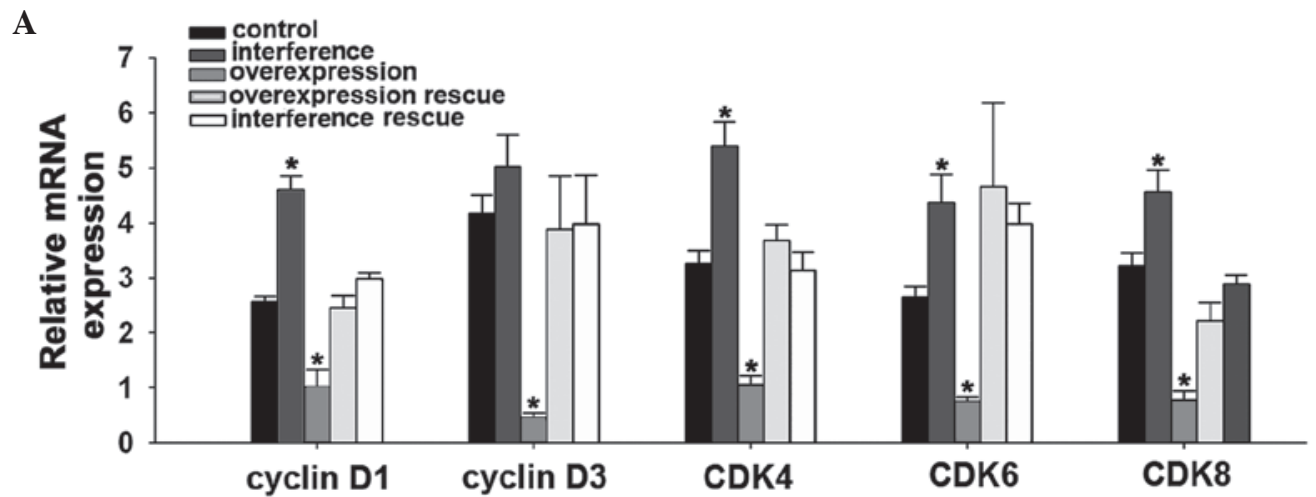

B

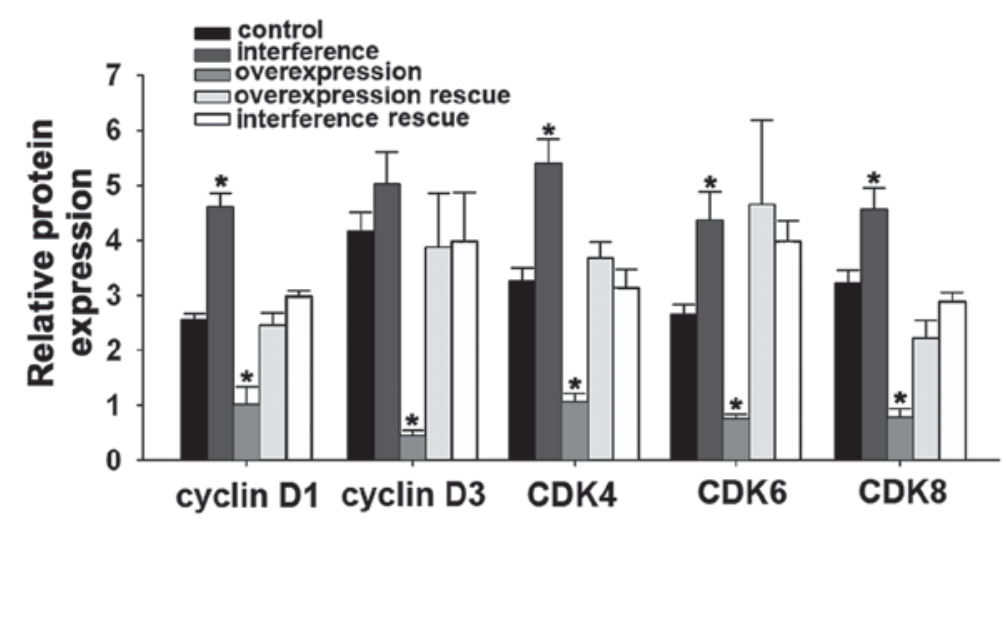

Figure 4. PIAsxa depresses cyclin D and CDK in U2-OS cells. (A) Reverse transcription-quantitative polymerase chain reaction analysis of cyclin D1, cyclin D3, CDK4, CDK6 and CDK8 in the control, interference, overexpression, overexpression rescue and interference rescue groups. (B) Western blot analysis of cyclin D1, cyclin D3, CDK4, CDK6 and CDK8 in the control, interference, overexpression, overexpression rescue and interference rescue groups. Data are presented as the mean \pm standard deviation. ${ }^{*} \mathrm{P}<0.05$, compared with the control. PIAsx $\alpha$, protein inhibitor of activated STAT x $\alpha$; CDK, cyclin D kinase.
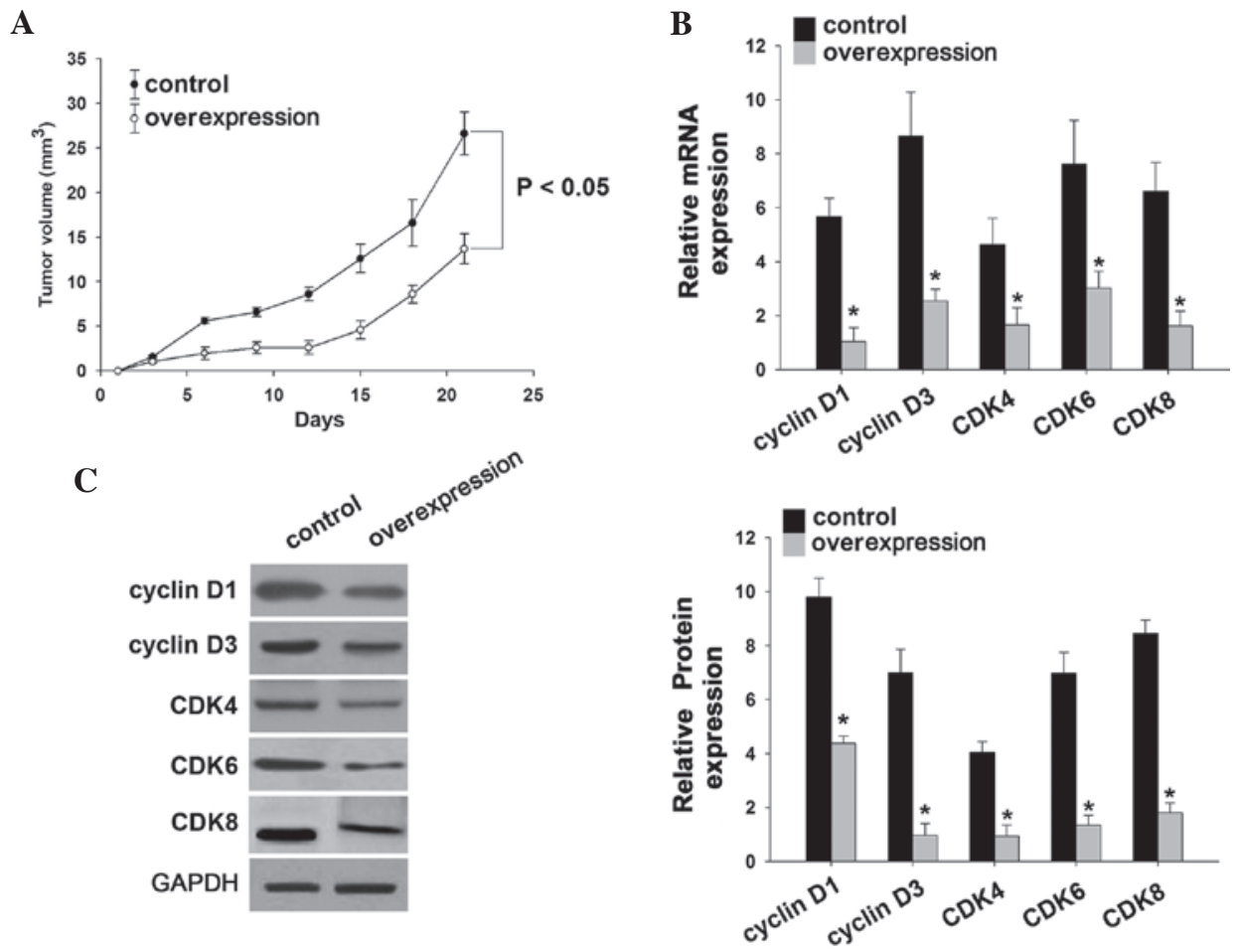

Figure 5. Inhibition of PIAsx $\alpha$ promotes tumor formation in a nude mouse tumor model. (A) Tumor volumes in the nude mouse model treated with U2-O2 cells overexpressing PIAsx $\alpha$ and untreated U2-OS cells. (B) Reverse transcription-quantitative polymerase chain reaction and (C) western blot analyses indicated that overexpression of PIAsx $\alpha$ decreased the expression levels of CDK2 and cyclin A in tumor tissues. Data are presented as the mean \pm standard deviation. ${ }^{*} \mathrm{P}<0.05$, compared with the control. PIAsx $\alpha$, protein inhibitor of activated STAT x $\alpha$; CDK, cyclin D kinase. 


\section{Discussion}

The results of the present study demonstrated that the inhibitory effect of PIAsx $\alpha$ on the progression of osteosarcoma cells occurred via the cyclin D and CDK pathway. The expression levels of PIAsx $\alpha$ in osteosarcoma tissues were markedly lower, compared with those in normal tissues, indicating impairment of the mechanism mediating the formation of osteosarcoma. In addition, the present study determined the expression levels of PIAsx $\alpha$ in different groups, including control, overexpression, interference, overexpression rescue and interference rescue groups. Of note, the overexpression of PIAsx $\alpha$ induced the inhibition of osteosarcoma cells, which is similar to other types of cancer cell, including hepatic carcinoma cells and colon cancer cells (27-29). In previous studies, it has been confirmed the downregulated expression levels of PIAsx $\alpha$ may be associated with tumor development and cell proliferation (29). Rodriguez et al reported that PIAsx $\alpha$ affects the expression of sumoylate PML and PML-RARA, affecting tumor development (30). Shinbo et al also found that PIAsxa is essential for PML degradation in lung carcinoma cells (31). These results are similar to those of the present study and, together, these findings suggest that PIAsx $\alpha$ is crucial in the regulation of oncogenic networks in tumor cells.

Following elucidation of the inhibitory effect of high expression levels of PIAsxa on osteosarcoma cells, the metastatic and invasive activities of the cells following changes in the expression of PIAsx $\alpha$ and changes in the cell cycle require investigation. At present, reports that PIAsx $\alpha$ alters the cell cycle in tumor cells directly are limited. Wang et al reported that overexpression of PIAsx $\alpha$ arrests the cell cycle in HeLa cells via a PTEN-dependent pathway (21). In the present study, the results indicated that, following overexpression of PIAsx $\alpha$, aptoptosis of the osteosarcoma was induced. It appears that downregulated expression levels of PIAsx $\alpha$ can control cell proliferation in cancer cells. Notably, the present study observed no differences in the overexpression rescue group, compared with the control group. In addition, the PIAsx $\alpha$ overexpression group arrested the cells at the G2/M stage. This is in accordance with the apoptotic effect of PIAsx $\alpha$ on osteosarcoma cells, which suggested that increased expression of PIAsx $\alpha$ may induce osteosarcoma cell apoptosis via controlling the cell cycle. Furthermore, these effects indicated that the progression between the $\mathrm{S}$ phase and $\mathrm{G} 2$ phase was inhibited by PIAsx $\alpha$ during mitosis. Taken together, the increased expression of PIAsx $\alpha$ triggered stagnancy of osteosarcoma cell proliferation at the $\mathrm{G}_{2} / \mathrm{M}$ stage, and progression of the cell cycle into mitosis resulted in the failure of cell division. This suggested that PIAsx $\alpha$ inhibited the progression of osteosarcoma cells, and has a regulatory role in cell cycle. However, the mechanisms underlying the effects of PIAsx $\alpha$ on the cell cycle in osteosarcoma cells require elucidation.

The expression levels of cyclin D1 and cyclin D3, and CDK4, CDK6 and CDK8 factors in the control, overexpression, interference, overexpression rescue and interference rescue groups were determined to understand the mechanism underlying the effect of PIAsx $\alpha$ on the inhibition of osteosarcoma cell progression. The results indicated that all the assessed factors decreased significantly in the overexpression group. It has been suggested that cyclin D1 can control cancer cell division by regulating transcription, mRNA amplification and stabilization (22). Similarly, cyclin D3 is also key in stimulating cell progression, particularly in cancer cells (22). The results of the present study showed that upregulated PIAsxa suppressed the expression of cyclin D1 and cyclin D3, at the mRNA and protein levels. The CDK factors, including CDK4, CDK6 and CDK8, were also depressed by upregulated PIAsx $\alpha$ at the mRNA and protein levels. CDK4, CDK6 and CDK8 have been confirmed as cell cycle modulators via the regulation of DNA synthesis and the progression between the G1 and $\mathrm{S}$ stages $(22,25)$. These factors also have been confirmed to be involved in several types of cancer, which indicates a potential therapeutic strategy. In the present study, PIAsx $\alpha$ depressed the expression levels of CDK4, CDK6 and CDK8 in vivo and in vitro. This suggested that the overexpression of PIAsx $\alpha$ in osteosarcoma cells suppresses the expression of cyclin D and CDK, offering a potential novel clinical therapy for osteosarcoma. In addition, the present study demonstrated that the upregulated expression of PIAsx $\alpha$ depressed tumor formation in a nude mouse model, also indicating PIAsx $\alpha$ as a potential therapeutic target in the treatment of osteosarcoma.

In conclusion, the present results demonstrated that, by upregulating the expression of PIAsx $\alpha$, osteosarcoma cells were suppressed and arrested at the G2/M stage. The molecular mechanism underlying these effects include the high expression levels of PIAsx $\alpha$ depressing cyclin D and CDK factors. Therefore, the expression of PIAsx $\alpha$ controls the expression levels of cyclin D and CDKs in cell proliferation. These findings offer novel insight for sumoylation and cell cycle in osteosarcoma, which may contribute to the treatment of patients with osteosarcoma.

\section{Acknowledgements}

This study was supported by the National Natural Science Foundation of China (grant. no. 81272947).

\section{References}

1. Geiss-Friedlander R and Melchior F: Concepts in sumoylation: A decade on. Nat Rev Mol Cell Biol 8: 947-956, 2007.

2. Yeh ET: SUMOylation and De-SUMOylation: Wrestling with life's processes. J Biol Chem 284: 8223-8227, 2009.

3. Saitoh H and Hinchey J: Functional heterogeneity of small ubiquitin-related protein modifiers SUMO-1 versus SUMO-2/3. J Biol Chem 275: 6252-6258, 2000.

4. Johnson ES: Protein modification by SUMO. Annu Rev Biochem 73: 355-382, 2004.

5. Owerbach D, McKay EM, Yeh ET, Gabbay KH and Bohren KM: A proline-90 residue unique to SUMO-4 prevents maturation and sumoylation. Biochem Biophys Res Commun 337: 517-520, 2005.

6. Mukhopadhyay D and Dasso M: Modification in reverse: The SUMO proteases. Trends Biochem Sci 32: 286-295, 2007.

7. Cheng J, Wang D, Wang Z and Yeh ET: SENP1 enhances androgen receptor-dependent transcription through desumoylation of histone deacetylase 1. Mol Cell Biol 24: 6021-6028, 2004.

8. Cheng J, Bawa T, Lee P, Gong L and Yeh ET: Role of desumoylation in the development of prostate cancer. Neoplasia 8: 667-676, 2006

9. Wang Q, Xia N, Li T, Xu Y, Zou Y, Zuo Y, Fan Q, Bawa-Khalfe T, Yeh ET and Cheng J: SUMO-specific protease 1 promotes prostate cancer progression and metastasis. Oncogene 32: 2493-2498, 2013.

10. Zhong S, Salomoni P and Pandolfi PP: The transcriptional role of PML and the nuclear body. Nat Cell Biol 2: E85-E90, 2000.

11. Moschos SJ and Mo YY: Role of SUMO/Ubc9 in DNA damage repair and tumorigenesis. J Mol Histol 37: 309-319, 2006. 
12. Alarcon-Vargas D and Ronai Z: SUMO in cancer-wrestlers wanted. Cancer Biol Ther 1: 237-242, 2002.

13. Mo YY, Yu Y, Theodosiou E, Ee PR and Beck WT: A role for Ubc9 in tumorigenesis. Oncogene 24: 2677-2683, 2005.

14. Zhu S, Sachdeva M, Wu F, Lu Z and Mo YY: Ubc9 promotes breast cell invasion and metastasis in a sumoylation-independent manner. Oncogene 29: 1763-1772, 2010.

15. Liu Lm, Yan Mg, Yang Dh, Sun WW and Zhang JX: PIAS3 expression in human gastric carcinoma and its adjacent non-tumor tissues. Clin Res Hepatol Gastroenterol 35: 393-398, 2011.

16. Okumura F, Matsunaga Y, Katayama Y, Nakayama KI and Hatakeyama S: TRIM8 modulates STAT3 activity through negative regulation of PIAS3. J Cell Sci 123: 2238-2245, 2010.

17. Li H, Gao H, Bijukchhe SM, Wang Y and Li T: PIAS3 may represent a potential biomarker for diagnosis and therapeutic of human colorectal cancer. Med Hypotheses 81: 1151-1154, 2013.

18. Stehmeier $\mathrm{P}$ and Muller S: Regulation of $\mathrm{p} 53$ family members by the ubiquitin-like SUMO system. DNA Repair (Amst) 8: 491-498, 2009

19. Palvimo JJ: PIAS proteins as regulators of small ubiquitin-related modifier (SUMO) modifications and transcription. Biochem Soc Trans 35: 1405-1408, 2007.

20. Jackson PK: A new RING for SUMO: Wrestling transcriptional responses into nuclear bodies with PIAS family E3 SUMO ligases. Genes Dev 15: 3053-3058, 2001.

21. Wang W, Chen Y, Wang S, Hu N, Cao Z, Wang W, Tong T and Zhang X: PIASxa ligase enhances SUMO1 modification of PTEN protein as a SUMO E3 ligase. J Biol Chem 289: 3217-3230, 2014.

22. Hunter T and Pines J: Cyclins and cancer II: Cyclin D and CDK inhibitors come of age. Cell 79: 573-582, 1994.
23. Sherr CJ: G1 phase progression: Cycling on cue. Cell 79: 551-555, 1994.

24. Association WM: World Medical Association Declaration of Helsinki: Ethical principles for medical research involving human subjects. Bulletin of the World Health Organization 79: 373, 2001.

25. Neuveut C, Low KG, Maldarelli F, Schmitt I, Majone F, Grassmann R and Jeang KT: Human T-cell leukemia virus type 1 Tax and cell cycle progression: Role of cyclin D-cdk and p110Rb. Mol Cell Biol 18: 3620-3632, 1998.

26. Livak KJ and Schmittgen TD: Analysis of relative gene expression data using real-time quantitative PCR and the 2(-Delta Delta C(T)) Method. Methods 25: 402-408, 2001.

27. Klampfer L: Signal transducers and activators of transcription (STATs): Novel targets of chemopreventive and chemotherapeutic drugs. Curr Cancer Drug Targets 6: 107-121, 2006.

28. Klampfer L: The role of signal transducers and activators of transcription in colon cancer. Front Biosci 13: 2888-2899, 2008.

29. Valentino L and Pierre J: JAK/STAT signal transduction: Regulators and implication in hematological malignancies. Biochem Pharmacol 71: 713-721, 2006.

30. Rodriguez MS, Desterro JM, Lain S, Midgley CA, Lane DP and Hay RT: SUMO-1 modification activates the transcriptional response of p53. EMBO J 18: 6455-6461, 1999.

31. Shinbo Y, Niki T, Taira T, Ooe H, Takahashi-Niki K, Maita C, Seino C, Iguchi-Ariga SM and Ariga H: Proper SUMO-1 conjugation is essential to DJ-1 to exert its full activities. Cell Death Differ 13: 96-108, 2006. 